\title{
A radiation dose review for paediatric fluoroscopy in an academic South African referral hospital
}

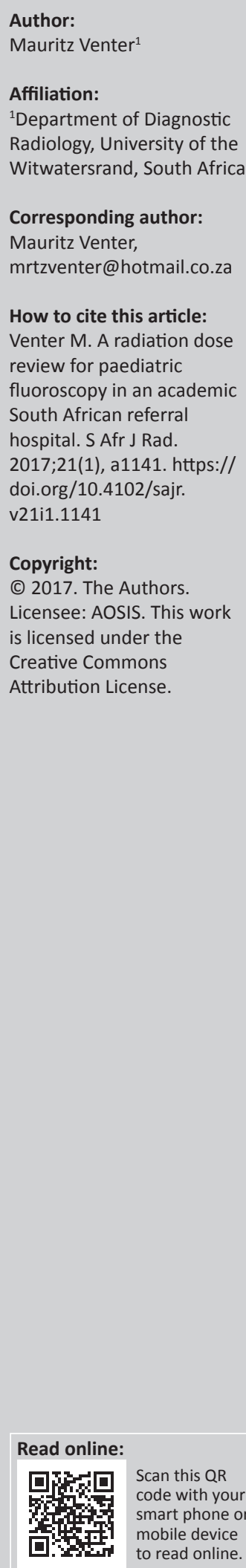

Background: It is important to reduce radiation exposure in children. There are no published South African paediatric fluoroscopic dosage references.

Aim: This study aims to determine the dose area product (DAP) values in children undergoing common fluoroscopic examinations and comparing the values to international standards. Commonly used examinations are contrasted swallows, contrasted enemas and vesicourethrograms (VCUs). The primary endpoint of this study will be the comparison of the median and third quartile DAP values for the four specified age groups to the values of the National UK Radiological Protection Board.

Method: We adhere to the Radiological Society of South Africa (RSSA)/South African Society of Paediatric Imaging's (SASPI) guidelines. The third quartile and mean DAP values were collected between March 2013 and March 2016 for each study performed, categorised into four age groups ( 0 year -1 year, 2 years -5 years, 6 years -10 years and 11 years -16 years) and stratified by our three major examinations. The data were compared to literature from the National UK Radiological Protection Board.

Results: DAP values for contrasted swallows were significantly lower in the three youngest age groups. There was no significant difference in the oldest age group. DAP values for VCUs were significantly lower in the youngest age group. There was no significant difference in the other three age groups. For our contrasted enemas, there were no data to compare with.

Conclusion: By following the SASPI guidelines, our overall DAP values compared better than the UK National Patient Dose Database values in the younger age groups and no worse in the older age groups.

Note: A selection of conference abstracts: RSSA/SASPI Paediatric Imaging Congress, 03-06 November 2016, Spier Estate, Stellenbosch, South Africa. Faculty collaborators: Professor Kassa Darge (Body Imaging, University of Pennsylvania, Philadelphia, USA), Professor Edward Lee (Thoracic Imaging, Harvard University, USA), Professor Beverley Newman (Cardiac Imaging, Stanford University, California, USA), Professor Kimberly Applegate (Image Gently and Body Imaging, Emory University, Atlanta, USA) and Professor Savvas Andronikou (Thoracic Imaging, University of Bristol, UK) supported by South African Paediatric Radiologists, co-ordinated by Dr Jaishree Naidoo, President of the African Society of Paediatric Imaging and Head of Division of Paediatric Radiology, Charlotte Maxeke Johannesburg Academic Hospital. 